\title{
DEEP LEARNING: A REVIEW
}

\author{
Rocio Vargas ${ }^{1}$, Amir Mosavi ${ }^{2,3^{*}}$, Ramon Ruiz ${ }^{1}$ \\ ${ }^{1}$ Obuda University, Faculty of Mechanical and Safety Engineering, 1081 Budapest, Hungary \\ ${ }^{2}$ Institute of Structural Mechanics, Bauhaus University Weimar, Weimar, Germany \\ ${ }^{3}$ Obuda University, Faculty of Electrical Engineering, 1034 Budapest, Hungary \\ Emails: amir.mosavi \& Rocio.Vargas \& Ramon.Ruiz@kvk.uni-obuda.hu
}

\begin{abstract}
Deep learning is an emerging area of machine learning (ML) research. It comprises multiple hidden layers of artificial neural networks. The deep learning methodology applies nonlinear transformations and model abstractions of high level in large databases. The recent advancements in deep learning architectures within numerous fields have already provided significant contributions in artificial intelligence. This article presents a state of the art survey on the contributions and the novel applications of deep learning. The following review chronologically presents how and in what major applications deep learning algorithms have been utilized. Furthermore, the superior and beneficial of the deep learning methodology and its hierarchy in layers and nonlinear operations are presented and compared with the more conventional algorithms in the common applications. The state of the art survey further provides a general overview on the novel concept and the ever-increasing advantages and popularity of deep learning.
\end{abstract}

Keywords: Deep learning, Machine Learning, Applied Deep Learning, Survey, Literature Review

\section{Introduction}

Artificial intelligence (AI) as an intelligence exhibited by machines has been an effective approach to human learning and reasoning [1]. In 1950, "The Turing Test" was proposed as a satisfactory explanation of how a computer could perform a human cognitive reasoning [2]. As a research field, AI is divided in more specific research subfields. For example: Natural Language Processing (NLP) [3] can enhance the writing experience in various applications $[4,17]$. The most classic subdivision within NLP is machine translation, which is understood as the translation between languages. Machine translation algorithms have resulted in various applications that consider grammar structure as well as spelling mistakes. Moreover, a set of words and vocabulary related to the main topic is automatically used as the main source when the computer is suggesting changes to writer or editor [5]. Fig. 1 shows in detail how AI covers seven subfields of computer sciences.

Recently, machine learning and data mining have become the center of attention and the most popular topics among research community. These combined fields of study 
analyze multiple possibilities of characterization of databases [9]. Through the years, databases have been collected with statistical purposes. Statistical curves can describe past, and present in order to predict future behaviors. Nevertheless, during the last decades only classic techniques and algorithms have been used to process this data, whereas an optimization of those algorithms could lead on an effective self-learning [19]. A better decision making can be implemented based on existing values, multiple criteria and statistics advanced methods. Thus, one of the most important application of this optimization is medicine, where symptoms, causes and medical solutions gen- erate big databases that can be used to predict better treatments [11].

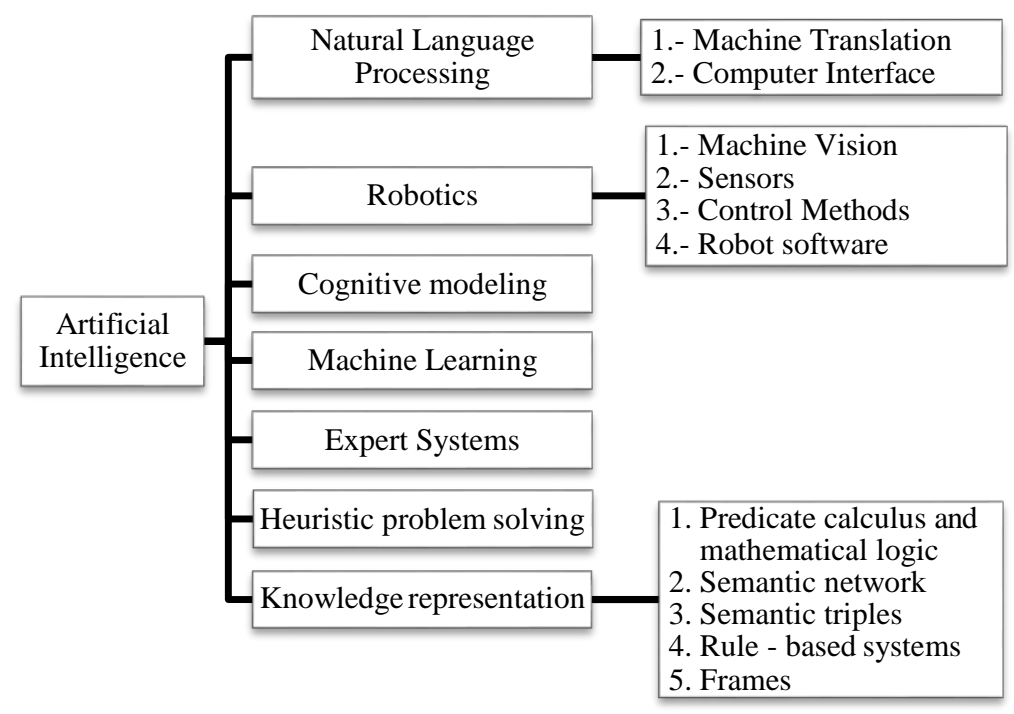

Fig. 1. Research in artificial intelligence (AI) Source: [1].

Since ML covers a wide range of research, many approaches have been established. Clustering, Bayesian Network, Deep Learning and Decision Tree Learning are only part of the approaches. The following review mainly focuses on deep learning, its basic concepts, past and nowadays applications in different fields. Additionally, it presents several figures portraying the rapid development of deep learning research through publications over the recent years in scientific databases.

\section{Background}

The Deep Learning (DL) concept appeared for the first time in 2006 as a new field of research within machine learning. It was first known as hierarchical learning at the [2], 
and it usually involved many research fields related to pattern recognition. Deep learning mainly considers two key factors: nonlinear processing in multiple layers or stages and supervised or unsupervised learning [4]. Nonlinear processing in multiple layers refers to an algorithm where the current layer takes the output of the previous layer as an input. Hierarchy is established among layers to organize the importance of the data to be considered as useful or not. On the other hand, supervised and unsupervised learning is related with the class target label, its availability means a supervised system, whereas its absence means an unsupervised system.

\section{Applications}

Deep learning implies an abstract layer analysis and hierarchical methods. However, it can be utilized in numerous real life applications. As an example, within digital image processing; gray scale image coloring from a picture used to be done manually by users who had to choose each color based on their own judgment. Applying a deep learning algorithm, coloring can be performed automatically by a computer [10]. Similarly, sound can be added into a mute drumming video by using Recurrent Neural Networks (RNN) as part of the deep learning methods [18].

Deep learning can be understood as a method to improve results and optimize processing times in several computing processes. In the field of natural language processing, deep learning methods have been applied for image caption generation [20], and handwriting generation [6]. The following applications are categorized in pure digital image processing, medicine and biometrics.

\subsection{Image Processing}

Before deep learning officially appeared as a new research approach, some applications had been carried out within the concept of pattern recognition through layer processing. In 2003, an interesting example was developed by applying particle filtering and Bayesian - belief propagation. The main concept of this application proposes that a human can recognize the face of a person by watching only a half - cropped face picture [14], therefore a computer could be able to reconstruct the image of a face from a cropped one.

Later in 2006, greed algorithm and hierarchy were combined into an application capable to process handwritten digits [7]. Recent researches have applied deep learning as the main tool for digital image processing. For instance, applying a Convolutional Neural Networks (CNN) for Iris Recognition can be more effective than using conventional iris sensors. CNN effectiveness can reach up to $99.35 \%$ of accuracy [16].

Mobile location recognition nowadays allows the user to know a determined address based on a picture. A Supervised Semantics - Preserving Deep Hashing (SSPDH) algorithm has proved a considerable improvement in comparison with Visual Hash Bit 
(VHB) and Space - Saliency Fingerprint Selection (SSFS). The accuracy of SSPDH is even $70 \%$ more efficient [15].

Finally, another remarkable application in digital image processing, using deep learning method, is facial recognition. Google, Facebook and Microsoft have unique deep learning face recognition models [8]. Lately, identification based on a facial picture has changed to an automatic recognition by establishing age and gender as initial parameters. Sighthound Inc. for instance tested a deep convolutional neural network algorithm capable to recognize not only age and gender, but even emotions [3]. Furthermore, a robust system was developed to accurately determine the age and gender of a person from a single image by the application of a deep multi-task learning architecture [21].

\subsection{Medicine}

Digital image processing is undeniably an important part of the research fields where a deep learning method can be applied. Thus, clinical applications have been recently tested. For instance, a comparison between shallow learning and deep learning in neural networks leaded to a better performance on disease prediction. An image taken from a Magnetic Resonance Imaging (MRI) [22] from a human brain was processed in order to predict a possible Alzheimer disease [3]. Although the early success of this procedure, some issues should be considered for future applications. Training and dependency on high quality are some of the limitations. Volume, quality and complexity of data are challenging aspects, however the integration of heterogeneous data types is a potential aspect of deep learning architecture [17, 23].

Optical Coherence Tomography (OCT) is another example where deep learning methods are showing valuable results. Conventionally, images are treated by a manual development of convolutional matrices [12]. Unfortunately, the lack of training sets, limits the deep learning method. Nevertheless within a few years, the introduction of better training sets will effectively predict retinal pathologies and decrease OCT technology costs [24].

\subsection{Biometrics}

In 2009, an automatic speech recognition application was carried out to decrease the Phone Error Rate (PER) by using two different architectures of deep belief networks [18]. In 2012, CNN [25] method was applied within the framework of a Hybrid Neural Network - Hidden Markov Model (NN - HMM). As a result, a PER of $20.07 \%$ was achieved. The PER obtained is better in comparison with a 3 - layer neural network baseline method previously applied [26]. Smartphones and their camera resolution have been tested on iris recognition. Using mobile phones developed by different companies the iris recognition accuracy can reach up to $87 \%$ of effectiveness [22,28]. 
Rocio Vargas, Amir Mosavi, Ramon Ruiz, Deep Learning: A Review, Advances in Intelligent Systems and

In terms of security, especially access control; deep learning is used in conjunction with biometric characteristics. DL was employed to speed up the developing and optimization of FaceSentinel face recognition devices. According to this manufacturer, their devices could expand their identification process from one-to-one to one-tomany in nine months [27]. This engine advancement could have taken 10 man years without DL introduction. It accelerated the production and launch of the equipment. These devices are used in Heathrow airport in London and have the potential to be used for time and attendance and in the banking sector [3, 29].

\section{Overview}

Table 1 summarizes several applications carried out throughout previous years regarding deep learning. Mostly, speech recognition and image processing are mentioned. This review only considers a few from the large list of applications.

Table 1. Deep Learning Applications, 2003 - 2017.

\begin{tabular}{|c|c|c|c|}
\hline Author & Application & Method/algorithm & Year \\
\hline $\begin{array}{l}\text { Tai Sing Lee, } \\
\text { David Mumford }\end{array}$ & $\begin{array}{l}\text { Hierarchical Bayesian in- } \\
\text { ference in the visual cor- } \\
\text { tex }\end{array}$ & $\begin{array}{l}\text { Particle filtering and } \\
\text { Bayesian - belief } \\
\text { propagation }\end{array}$ & 2003 \\
\hline Hinton, Geoffrey E., Simon & & Complementary Priors & \\
\hline $\begin{array}{l}\text { Osindero, } \\
\text { Yee-WhyeTeh. }\end{array}$ & Digit Classification & $\begin{array}{c}\text { on } \\
\text { Belief networks } \\
\end{array}$ & 2006 \\
\hline $\begin{array}{l}\text { Mohamed, Abdel-rahman, } \\
\text { George Dahl, } \\
\text { Geoffrey Hinton }\end{array}$ & $\begin{array}{l}\text { Deep Belief Networks } \\
\text { for phone recognition }\end{array}$ & $\begin{array}{c}\text { Back propagation and as- } \\
\text { sociative memory archi- } \\
\text { tecture }\end{array}$ & 2009 \\
\hline $\begin{array}{l}\text { Abdel-Hamid Ossama, Mo- } \\
\text { hamed Abdel-rahman,Jiang } \\
\text { Hui, } \\
\text { Penn Gerald }\end{array}$ & $\begin{array}{l}\text { Multi-speaker speech } \\
\text { recognition }\end{array}$ & $\begin{array}{l}\text { Local filtering and max- } \\
\text { pooling infrequency do- } \\
\text { main }\end{array}$ & 2012 \\
\hline $\begin{array}{l}\text { Kiran B. Raja, } \\
\text { R. Raghavendra, } \\
\text { Vinay Krishna Vemuri, } \\
\text { Christoph Busch }\end{array}$ & $\begin{array}{l}\text { Iris Recognition by using } \\
\text { smartphones' cameras }\end{array}$ & Deep sparse filtering & 2015 \\
\hline Silver David, et al & $\begin{array}{c}\text { Mastering the Game of } \\
\text { Go with Deep Neural } \\
\text { Networks and Tree } \\
\text { Search }\end{array}$ & $\begin{array}{l}\text { Supervised learning and } \\
\text { reinforcement learning }\end{array}$ & 2016 \\
\hline $\begin{array}{l}\text { Francesco Marra, Giovanni } \\
\text { Poggi, } \\
\text { Carlo Sansone, }\end{array}$ & $\begin{array}{l}\text { Iris sensor model identi- } \\
\text { fication }\end{array}$ & $\begin{array}{c}\text { Convolutional neural } \\
\text { networks }\end{array}$ & 2017 \\
\hline
\end{tabular}


Rocio Vargas, Amir Mosavi, Ramon Ruiz, Deep Learning: A Review, Advances in Intelligent Systems and

Luisa Verdoliva

\subsection{Publication analysis per year}

Fig. 1 details the number of deep learning publications from ScienceDirect database per year from 2006 till June 2017. Clearly, there is a progressive increment of publications that could describe an exponential growth.

Fig. 2 presents the total number of Springer's deep learning publications per year from Jan 2006 till Jun 2017. In 2016, there is a sudden increment of publications, reaching up to 706 publications, which proves that deep learning is truly a contemporaneous area of interest for researchers.

Fig. 3 shows the IEEE Digital Library's number of conference publications and journals \& magazines from Jan 2006 till the Jun 2017. It is noticeable that since 2015 the number of publications has dramatically increased. The difference between 2016 and 2015 is even more than $200 \%$ of increment.

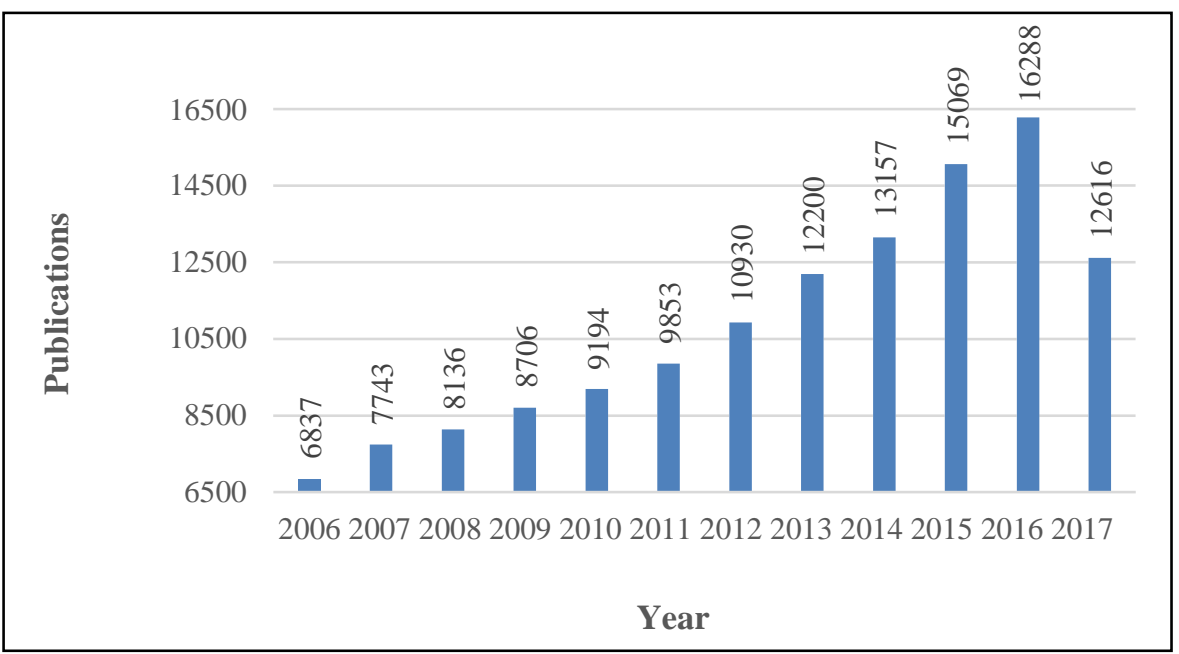

Fig. 1. Growth of the number of publications in Deep Learning, Sciencedirect database (Jan 2006-Jun 2017) 


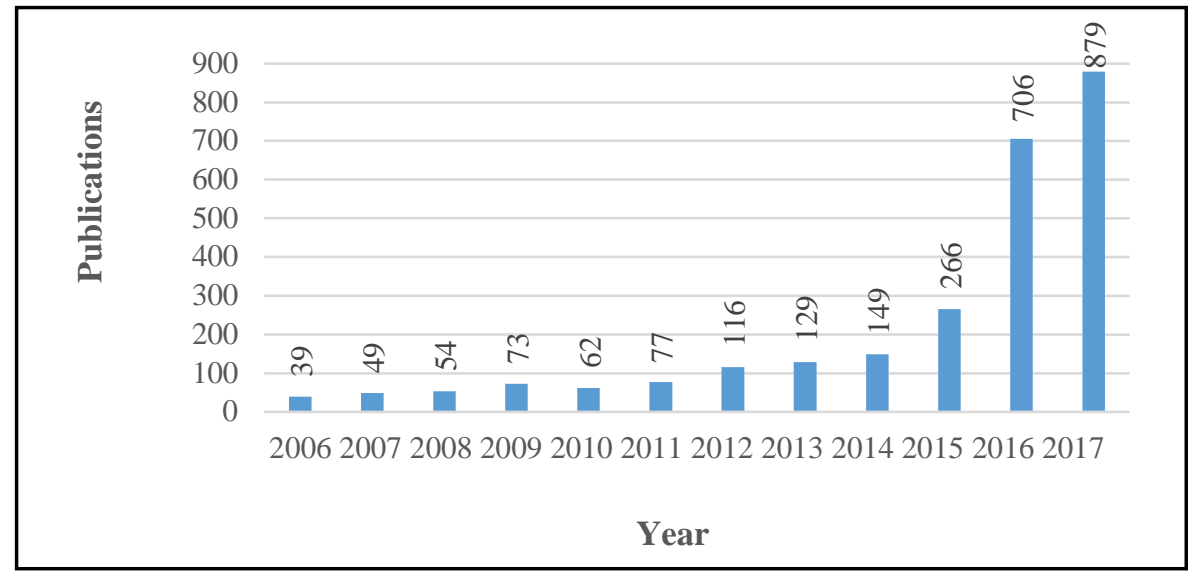

Fig. 2. Growth of the number of publications in Deep Learning from Springer database. (Jan 2006-Jun 2017)

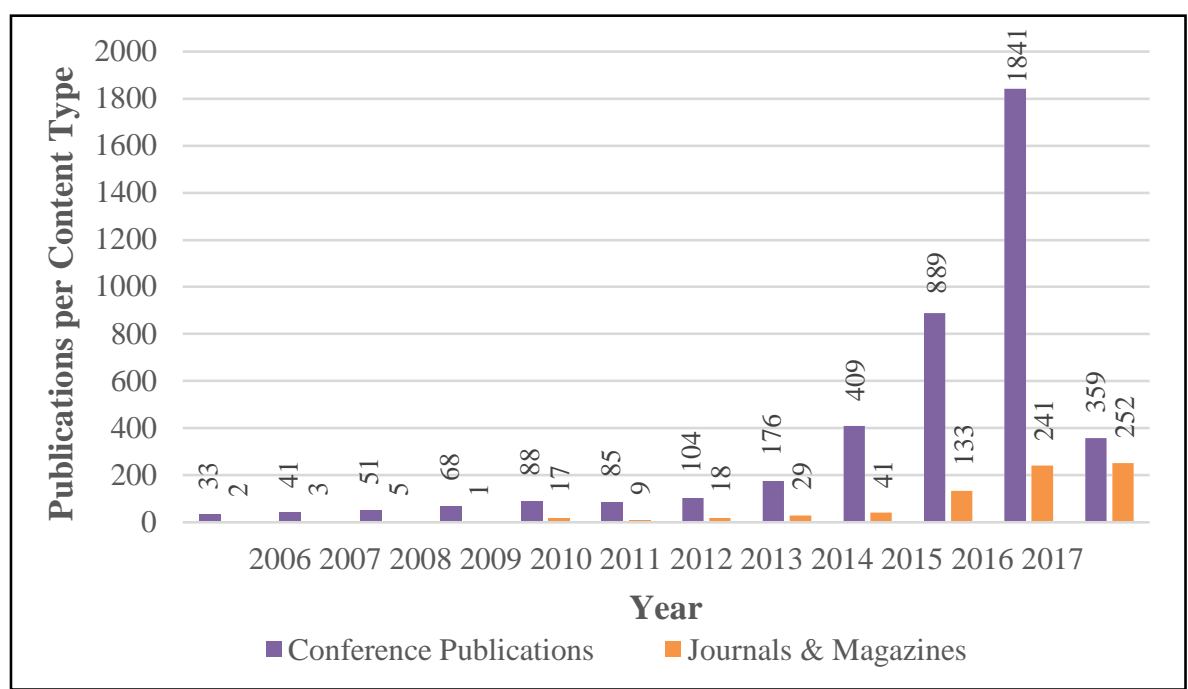

Fig. 3. Growth of publications in Deep Learning from IEEE database. (Jan 2006-Jun 2017)

\section{Conclusions}

Deep learning is indeed a fast growing application of machine learning. The numerous applications described above prove its rapid development in just few years. The usage of these algorithms in different fields shows its versatility. The publication analysis 
performed in this study clearly demonstrates the relevance of this technology and gives a clearly illustrates the growth of deep learning and the tendency regarding for future research in this field.

Additionally, it is important to note that hierarchy of layers and the supervision in learning are key factors to develop a successful application regarding deep learning. Hierarchy is essential for appropriate data classification, whereas supervision considers the importance of the database itself to be part of the process. The main value of deep learning relies on the optimization of existing applications in machine learning, due to its innovativeness on hierarchical layer processing. Deep learning can deliver effective results on digital image processing and speech recognition. The reduction on error percentage (10 to $20 \%$ ) clearly corroborates the improvement compared with existing and tested methods.

During the current era and in a future, deep learning can result into a useful security tool due to the facial recognition and speech recognition combined. Besides this, digital image processing is a research field that can be applied in multiple areas. For this reason and having proved a true optimization, deep learning is a contemporary and exciting subject of advancement in artificial intelligence.

\section{References}

1. Abdel, O.: Applying convolutional neural networks concepts to hybrid NN-HMM model for speech recognition. Acoustics, Speech and Signal Processing 7, 4277-4280 (2012).

2 Mosavi A., Varkonyi-Koczy A. R.: Integration of Machine Learning and Optimization for Robot Learning. Advances in Intelligent Systems and Computing 519, 349-355 (2017).

3. Bannister, A.: Biometrics and AI: how FaceSentinel evolves 13 times faster thanks to deep learning (2016).

4. Bengio, Y.: Learning deep architectures for AI. Foundations and trends in Machine Learning 2, 1-127 (2009).

5. Mosavi, A., Varkonyi-Koczy, A. R., Fullsack, M.: Combination of Machine Learning and Optimization for Automated Decision-Making. MCDM (2015).

6. Deng L, Yu D Deep learning: methods and applications. Foundations and Trends ${ }^{\circledR}$ in Signal Processing 7, 197-387 (2014)

7. Goel, B.: Developments in The Field of Natural Language Processing. International Journal of Advanced Research in Computer Science 8, (2017).

8. Vaezipour, A.: Mosavi, A. Seigerroth, U.: Machine learning integrated optimization for decision making, 26th Europian Conference on Operational Research, Rome (2013).

9. Hinton G E, Simon O, Yee-Whye T A fast learning algorithm for deep belief nets. Neural computation 18, 1527-1554 (2006)

10. Hisham, A., Harin, S.: Deep Learning - the new kid in Artificial Intelligence. (2017)

11. Kim I W, Oh, M.: Deep learning: from chemoinformatics to precision medicine. Journal of Pharmaceutical Investigation: 1-7 (2017)

12. Mosavi, A., Vaezipour, A.: Developing Effective Tools for Predictive Analytics and Informed Decisions. Technical Report. University of Tallinn (2013) 
13. Mosavi A., Y., Bathla, Varkonyi-Koczy A. R.: Predicting the Future Using Web Knowledge: State of the Art Survey Advances in Intelligent Systems and Computing (2017).

14. Mosavi, A., Vaezipour, A.: Reactive Search Optimization; Application to Multiobjective Optimization Problems. Applied Mathematics 3, 1572-1582 (2012)

15. Lee J-G (2017) Deep Learning in Medical Imaging: General Overview. Korean Journal of Radiology 18(4):570-584

16. Lee T.: David M Hierarchical Bayesian inference in the visual cortex. JOSA 20, 1434-1448 (2003).

17. Liu W Deep learning hashing for mobile visual search. EURASIP Journal on Image and Video Processing 17, (2017).

18. Marra F.: A Deep Learning Approach for Iris Sensor Model Identification. Pattern Recognition Letters (2017).

19. Miotto R et al (2017) Deep learning for healthcare: review, opportunities and challenges. Briefings in Bioinformatics

20. Mohamed A.: Deep belief networks for phone recognition. Nips workshop on deep learning for speech recognition and related applications: 1, 635-645 (2009).

21. Moor. J.: The Turing test: the elusive standard of artificial intelligence. Springer Science \& Business Media (2003).

22. Vaezipour, A. Mosavi, U. Seigerroth, A.: Visual analytics and informed decisions in health and life sciences, International CAE Conference, Verona, Italy (2013)

23. Raja K B, Raghavendra R, Vemuri V K, Busch C (2015) Smartphone based visible iris recognition using deep sparse filtering. Pattern Recognition Letters 57:33-42.

24. Safdar S, Zafar S, Zafar N, Khan N F (2017) Machine learning based decision support systems (DSS) for heart disease diagnosis: a review. Artificial Intelligence Review: 1-17

25. Mosavi, A. Varkonyi. A.: Learning in Robotics. Learning 157, (2017)

26. Xing J, Li K, Hu W, Yuan C, Ling H et al (2017) Diagnosing deep learning models for high accuracy age estimation from a single image. Pattern Recognition

27. Mosavi, A. Rabczuk, T.: Learning and Intelligent Optimization for Computational Materials Design Innovation, Learning and Intelligent Optimization, Springer-Verlag, (2017)

28. Vaezipour, A., et al., Visual analytics for informed-decisions, International CAE Conference, Verona, Italy, (2013).

29. Dehghan, A.: DAGER: Deep Age, Gender and Emotion Recognition Using Convolutional Neural Network3, 735-748 (2017)

30. Mosavi, A: predictive decision model, 2015, https://doi.org/10.13140/RG.2.2.21094.630472

31. Vaezipour, A., et al.: Visual analytics and informed decisions in health and life sciences." Paper in Proceedings of International CAE Conference, Verona, Italy. (2013).

32. Vaezipour, A.: Visual analytics for informed-decisions, CAE Conference, Italy, (2013).

33. A.Vaezipour, A.:Machine learning integrated optimization for decision making. 26th European Conference on Operational Research, Rome (2013).

34. Vaezipour, A.: Visual Analytics for Multi-Criteria Decision Analysis, in Proceedings of International CAE Conference, Verona, Italy (2013).

35. Mosavi, A., Vaezipour, A.: Developing Effective Tools for Predictive Analytics and Informed Decisions. Technical Report. (2013). https://doi.org/10.13140/RG.2.2.23902.84800

36. Mosavi A., Varkonyi-Koczy A. R.: Integration of Machine Learning and Optimization for Robot Learning. Advances in Intelligent Systems and Computing 519, 349-355 (2017).

37. Mosavi, A., Varkonyi, A.: Learning in Robotics. Learning, 157, (2017). 
38. Mosavi, A.: Decision-making software architecture; the visualization and data mining assisted approach. International Journal of Information and Computer Science 3, 12-26 (2014).

39. Mosavi, A.: The large scale system of multiple criteria decision making; pre-processing, Large Scale Complex Systems Theory and Applications 9, 354-359 (2010).

40. Esmaeili, M., Mosavi, A.: Variable reduction for multiobjective optimization using data mining techniques. Computer Engineering and Technology 5, 325-333 (2010)

41. Mosavi, A.: Data mining for decision making in engineering optimal design. Journal of AI and Data Mining 2, 7-14 (2014).

42. Mosavi, A., Vaezipour, A.: Visual Analytics, Obuda University, Budapest, (2015).

43. Mosavi, A., Vaezipour, A.: Reactive Search Optimization; Application to Multiobjective Optimization Problems. Applied Mathematics 3, 1572-1582 (2012).

44. Mosavi, A., Varkonyi-Koczy, A. R., Fullsack, M.: Combination of Machine Learning and Optimization for Automated Decision-Making. MCDM (2015).

45. Mosavi, A., Delavar, A.: Business Modeling, Obuda University, Budapest, (2016).

46. Mosavi, A.: Application of data mining in multiobjective optimization problems. International Journal for Simulation and Multidisciplinary Design Optimization, 5, (2014)

47. Mosavi, A. Rabczuk, T.: Learning and Intelligent Optimization for Material Design Innovation, Theoretical Computer Science and General Issues, LION11 (2017).

48. Mosavi, A., Visual Analytics, Obuda University, 2016.

49. Mosavi, A.: Predictive decision making, Tech Rep 2015. doi: 10.13140/RG.2.2.16061.46561

50. Mosavi. A.: Predictive Decision Making, Predictive Decision Model, Tech. Report. (2015). https://doi.org/10.13140/RG.2.2.21094.63047

51. Mosavi, A., Lopez, A.: Varkonyi-Koczy, A.: Industrial Applications of Big Data: State of the Art Survey, Advances in Intelligent Systems and Computing, (2017).

52. Mosavi, A., Rabczuk, T., Varkonyi-Koczy, A.: Reviewing the Novel Machine Learning Tools for Materials Design, Advances in Intelligent Systems and Computing, (2017).

53. Mousavi, S., Mosavi, A., Varkonyi-Koczy, A. R.: A load balancing algorithm for resource allocation in cloud computing, Advances in Intelligent Systems and Computing, (2017).

54. Baranyai, M., Mosavi, A., Vajda, I., Varkonyi-Koczy, A. R.: Optimal Design of Electrical Machines: State of the Art Survey, Advances in Intelligent Systems and Computing, (2017).

55. Mosavi, A., Benkreif, R., Varkonyi-Koczy, A.: Comparison of Euler-Bernoulli and Timoshenko Beam Equations for Railway System Dynamics, Advances in Intelligent Systems and Computing, (2017).

56. Mosavi, A., Rituraj, R., Varkonyi-Koczy, A. R.: Reviewing the Multiobjective Optimization Package of modeFrontier in Energy Sector, Advances in Intelligent Systems and Computing, (2017).

57. Mosavi, A., Bathla, Y., Varkonyi-Koczy A. R.: Predicting the Future Using Web Knowledge: State of the Art Survey, Advances in Intelligent Systems and Computing, (2017). 